\title{
O ciclo glicose-ácido graxo durante exercício intenso: uma teoria contestável?
}

\author{
The glucose-fatty acid cycle during intense \\ exercise: a contestable theory?
}

Fábio Rosseto'

$\mathrm{E}^{\mathrm{n}}$ m apreciação ao estudo de Silveira e cols. (1), publicado em 2011 nos Arquivos Brasileiros de Endocrinologia e Metabologia, e de Philip Randle e cols. (2), a respeito da teoria clássica do ciclo glicose-ácido graxo, algumas considerações merecem nota. Em sua revisão a respeito da regulação do metabolismo da glicose e ácido graxo no músculo esquelético durante o exercício físico, Silveira e cols. (1) discutem a seguinte questão: Por que o ciclo glicose-ácido graxo falha em exercer seus efeitos durante o exercício intenso? De acordo com os autores, o fornecimento da oxidação de lipídeos (OL) e redução da oxidação de carboidratos (OCHO), efeito do ciclo da glicose-ácido graxo, explica o aumento da capacidade do músculo esquelético em sustentar atividades prolongadas (geralmente menos intensas), mas não em atividades intensas (maior OCHO). Similarmente, Jeukendrup (3) menciona não haver evidências que fundamentem o ciclo da glicose-ácido graxo relacionando a teoria aos exercícios de moderada a altas intensidades, normalmente descritas pela porcentagem do consumo máximo de oxigênio $\left(\mathrm{V}_{\text {2max }}\right)$.

Inicialmente, devemos pontoar que o ciclo glicose-ácido graxo estabelece a regulação metabólica crucial para a homeostase energética $(4,5)$. Fundamentar o efeito metabólico dos substratos durante o exercício comparativamente ao ciclo glicose-ácido graxo, que demanda tempo para que haja a regulação homeostática, descaracteriza a primazia da teoria em questão. Sob condições da vida cotidiana, Jequier (6) reporta que o balanço do carboidrato deve ser obtido dentro de 24 ou 48 horas, levando à premissa de que o balanço lipídico seja similar. A respeito da seleção metabólica do combustível (substratos), em outro trabalho Randle (7) menciona que a regulação da oxidação de glicose ocorre em longo prazo, resultado do efeito dos ácidos graxos na fosforilação reversível do complexo mitocondrial piruvato desidrogenase. Ao analisar o efeito de intensidades distintas de exercício físico sob a OL em 24-h, alguns estudos clínicos aleatórios reportam que não há diferença significativa entre as comparações (811) (Tabela 1). Treuth e cols. (12), ao analisarem a intensidade do exercício sob a oxidação dos substratos em 24-h, puderam verificar taxas semelhantes de OL e OCHO.

Em contraste com o estudo de Silveira e cols. (1), independente da intensidade do exercício, ao analisar a oxidação dos substratos sob um panorama de tempo mais amplo, em 24-h a OL é significantemente invariável e a OCHO (significativamente maior) reflete a diferença entre a OL e o gasto energético, mantendo a homeostase energética. Em resumo, a teoria de Randle e cols. (2) não parece ser falha ao considerarmos com fidelidade que o ciclo glicose-ácido graxo representa a regulação homeostática dos substratos sob uma análise de tempo mais longa, e, qualquer comparação que desconsidere esse período ( 24-h), em que ocorre a variação quantitativa da oxidação de ácido graxo e glicose, pode induzir a interpretações contestáveis.

Correspondência para: Fábio Rosseto Av. 31 de Março, 895, ap. 6 11740-000 - Itanhaém, SP, Brasil fabio.rosseto@yahoo.com.br

Recebido em 24/Jun/2012 Aceito em 6/Set/2012 
Tabela 1. 0 efeito do exercício em intensidades distintas sob a oxidação dos substratos em 24-h

\begin{tabular}{|c|c|c|c|c|c|}
\hline Estudo & Participantes & Intervenção & $\begin{array}{c}\text { Oxidação de } \\
\text { carboidrato } \\
\left(\mathbf{g} \cdot \mathbf{d}^{-1}\right)\end{array}$ & $\begin{array}{c}\text { Oxidação de lipídios } \\
\left(\mathbf{g} \cdot \mathrm{d}^{-1}\right)\end{array}$ & Resultados \\
\hline $\begin{array}{l}\text { Melanson e cols. } \\
(2002)(8)\end{array}$ & $\begin{array}{c}(\bar{x} \pm D P) \\
\text { N: } \\
10 \mathrm{H}, \mathrm{NOB} \\
\text { Idade: } \\
31 \pm 7\end{array}$ & $\begin{array}{c}(\bar{x} \pm E P) \\
\text { 1) } E A: 49 \pm 7 \text { min a } 70 \% \\
\dot{V} 0_{2 \operatorname{máx}} \\
\text { 2) ER em circuito: } 60 \text { min, } \\
4 \text { séries, } 10 \text { rep., } 70 \% \\
\text { 1RM, } 3 \text { min int. }\end{array}$ & $\begin{array}{c}(\bar{x} \pm E P) \\
\text { CON: } 249 \pm 29 \\
\text { EA: } 370 \pm 18 \\
\text { ER: } 349 \pm 23\end{array}$ & $\begin{array}{c}(\bar{x} \pm E P) \\
\text { CON: } 86 \pm 17 \\
\text { EA: } 87 \pm 12 \\
\text { ER: } 91 \pm 10\end{array}$ & $\begin{array}{c}\text { OCHO 24-h: } \\
\text { CON < EX } \\
(P=0,04) \\
\text { OL 24-h: } \\
\text { CON = EX } \\
\text { (P: NS) }\end{array}$ \\
\hline $\begin{array}{l}\text { Melanson e cols. } \\
(2002)(9)\end{array}$ & $\begin{array}{c}(\bar{x} \pm \mathrm{DP}) \\
\mathrm{N}: \\
8 \mathrm{H}, 8 \mathrm{M}, \mathrm{NOB} \\
\\
\text { Idade: } \\
30.4 \pm 7.6(\mathrm{H}) \\
29.5 \pm 5.1(\mathrm{M})\end{array}$ & 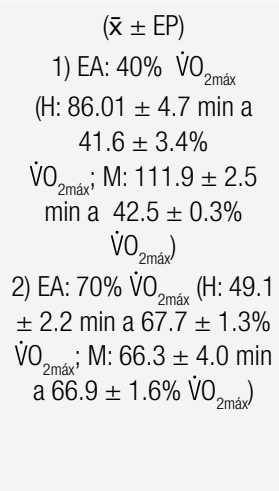 & 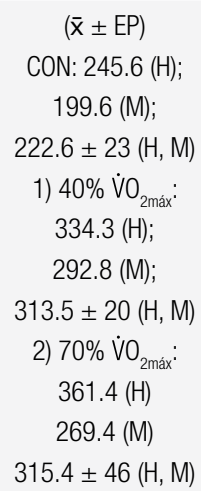 & $\begin{array}{c}(\bar{x} \pm \mathrm{EP}) \\
\text { CON: } 90.7(\mathrm{H}) ; 83.7(\mathrm{M}) ; \\
87.2 \pm 3.5(\mathrm{H}, \mathrm{M}) \\
\text { 1) } 40 \% \text { VO }{ }_{2 \text { máx }}: 96.3(\mathrm{H}) ; \\
90.7(\mathrm{M}) ; \\
93.5 \pm 2.8(\mathrm{H}, \mathrm{M}) \\
\text { 2) } 70 \% \dot{\mathrm{V}} \mathrm{O}_{2 \text { máx }}: \\
92.3(\mathrm{H}) ; \\
105.9(\mathrm{M}) ; \\
99.1 \pm 6.8(\mathrm{H}, \mathrm{M})\end{array}$ & $\begin{array}{c}\text { OCHO 24-h: } \\
\text { CON < EX } \\
\left(40=70 \% \text { VO }_{2 m a x x}\right) \\
(P: N S) \\
H>M \\
(P=0,035) \\
\text { OL 24-h: } \\
\text { CON }=E X \\
(40=70 \%) O_{2 m a x} \\
M>H \\
(P=0.02)\end{array}$ \\
\hline $\begin{array}{l}\text { Saris e cols. } \\
(2004)(10)\end{array}$ & $\begin{array}{c}(\overline{\mathrm{x}} \pm \mathrm{EP}) \\
\mathrm{N}: 8 \mathrm{H}, \mathrm{OB} \\
\text { Idade: } \\
38.3 \pm 1.1\end{array}$ & $\begin{array}{l}\text { 1) EA: } 3 \text { sessões de } \\
60 \text { min a } 38 \% W_{\text {máx }} \\
\text { 2) EA intervalado: } \\
3 \text { sessões de } 30 \text { min } \\
\text { alternados em ataques de } \\
2.5 \text { min a } 80 \text { e } 50 \% W_{\text {máx }}\end{array}$ & ND & ND & $\begin{array}{c}\text { OCHO 24-h: } \\
38=80 \text { e } 50 \% \mathrm{~W}_{\text {máx }} \\
\text { OL } 24-h: \\
38=80 \text { e } 50 \% \mathrm{~W}_{\text {máx }}\end{array}$ \\
\hline $\begin{array}{l}\text { Melanson e cols. } \\
(2006)(11)\end{array}$ & $\begin{array}{c}(\bar{x} \pm \mathrm{DP}) \\
\mathrm{N}: \\
15 \mathrm{OB}: \\
(7 \mathrm{H}, 8 \mathrm{M}) \\
11 \mathrm{NOB}: \\
(5 \mathrm{H}, 6 \mathrm{M}) \\
\text { Idade: } \\
35 \pm 10 \text { (H, OB) } \\
33 \pm 7(\mathrm{M}, \mathrm{OB}) \\
33 \pm 9 \text { (H, NOB) } \\
26 \pm 7 \text { (M, NOB) }\end{array}$ & $\begin{array}{l}\text { 1) } \mathrm{EA}: 60 \text { min a } 40 \% \\
\dot{\mathrm{V}} \mathrm{O}_{2 \operatorname{máx}} \\
\text { 2) } \mathrm{EA}: 30 \text { min a } 70 \% \\
\dot{\mathrm{V}} \mathrm{O}_{2 \operatorname{máx}}\end{array}$ & ND & $\begin{array}{c}(\bar{x} \pm E P) \\
\text { OB: } \\
\text { CON: } 90 \pm 13 \\
\text { 1) } E A: 79 \pm 16 \\
\text { 2) } E A: 86 \pm 15 \\
\text { NOB: } \\
\text { CON: } 92 \pm 13 \\
\text { 1) } E A: 92 \pm 16 \\
\text { 2) } E A: 73 \pm 15\end{array}$ & $\begin{array}{c}\text { OCHO 24-h: ND } \\
\text { OL 24-h: } \\
\text { CON }=\text { EX } \\
\left(40=70 \% \text { VO }_{2 \text { max }}\right) \\
\text { (P: NS) }\end{array}$ \\
\hline
\end{tabular}

N: número; H: homem; M: mulher; NOB: não obeso; OB: obeso; EX: exercício; EA: exercício aeróbio; ER: exercício resistido; CON: grupo controle; ( $\overline{\mathrm{X}} \pm$ DP): desvio-padrão da média apresentado no estudo; ( $\overline{\mathrm{X}} \pm$ EP): erro-padrão da média apresentado no estudo; $\mathrm{V}_{2}{ }_{\text {máx }}$ : volume máximo de oxigênio consumido; rep.: repetições; $1 \mathrm{RM}$ : uma repetição máxima; int.: intervalo; OL: oxidação lipídica; OCHO: oxidação de carboidrato; $\mathrm{W}_{\text {max }}$ : capacidade máxima de trabalho; NS: valores de $\mathrm{P}$ não significativos de acordo com os autores; ND: valores não descritos pelos autores.

Declaração: o autor declara não haver conflitos de interesse científico neste estudo.

\section{REFERÊNCIAS}

1. Silveira LR, Pinheiro CHdJ, Zoppi CC, Hirabara SM, Vitzel KF, Bassit RA, et al. Regulação do metabolismo de glicose e ácido graxo no músculo esquelético durante exercício físico. Arq Bras Endocrinol Metab. 2011;55:303-13.

2. Randle PJ, Garland PB, Hales CN, Newsholme EA. The glucose fatty-acid cycle. Its role in insulin sensitivity and the metabolic disturbances of diabetes mellitus. Lancet. 1963;1(7285):785-9.
3. Jeukendrup AE. Regulation of fat metabolism in skeletal muscle. Ann NY Acad Sci. 2002;967:217-35.

4. Sugden MC. In appreciation of Sir Philip Randle: the glucose-fatty acid cycle. Br J Nutr. 2007;97(5):809-13.

5. Hue L, Taegtmeyer H. The Randle cycle revisited: a new head for an old hat. Am J Physiol Endocrinol Metab. 2009;297(3):E578-91.

6. Jequier E. Nutrient effects: post-absorptive interactions. Proc Nutr Soc. 1995;54(1):253-65.

7. Randle PJ. Metabolic fuel selection: general integration at the whole-body level. Proc Nutr Soc. 1995;54(1):317-27.

8. Melanson EL, Sharp TA, Seagle HM, Donahoo WT, Grunwald GK, Peters JC, et al. Resistance and aerobic exercise have similar effects on 24-h nutrient oxidation. Med Sci Sports Exerc. 2002;34(11):1793-800. 
9. Melanson EL, Sharp TA, Seagle HM, Horton TJ, Donahoo WT, Grunwald GK, et al. Effect of exercise intensity on 24-h energy expenditure and nutrient oxidation. J Appl Physiol. 2002;92(3):1045-52.

10. Saris WH, Schrauwen P. Substrate oxidation differences between high- and low-intensity exercise are compensated over 24 hours in obese men. Int J Obes Relat Metab Disord. 2004;28(6):759-65.
11. Melanson EL, Cornier MA, Bessesen DH, Grunwald GK, MacLean PS, Hill JO. $24 \mathrm{H}$ metabolic responses to low- and high-intensity exercise in lean and obese humans. Obesity. 2006;14(1):180-2.

12. Treuth MS, Hunter GR, Williams M. Effects of exercise intensity on 24-h energy expenditure and substrate oxidation. Med Sci Sports Exerc. 1996;28(9):1138-43. 